\title{
EDITORIAL
}

\section{Beyond arterial remodelling: pulmonary venous and cardiac involvement in patients with systemic sclerosis- associated pulmonary arterial hypertension}

\author{
P. Dorfmüller*,\#, , D. Montanï,\#, and M. Humbert*,\#,
}

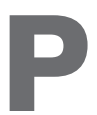

ulmonary arterial hypertension (PAH) represents a heterogeneous group of disorders characterised by elevated pulmonary vascular resistance and a normal pulmonary artery wedge pressure that occurs in the absence of left heart disease, chronic lung diseases/hypoxia or chronic thromboembolic pulmonary disease [1]. Entities within the PAH group share not only similar symptoms and haemodynamic profiles but also a common therapeutic approach [2]. Unfortunately, outcome among $\mathrm{PAH}$ patients remains poor and a cure for the disease remains elusive [3]. One additional, although less well described, feature that favours the distinction of a PAH group is the histomorphological correlate of elevated blood pressures within the pulmonary vasculature. Indeed, PAH that is idiopathic, heritable, or associated with anorexigen exposure, HIV infection, portopulmonary hypertension and connective tissue disease (comprising mostly systemic sclerosis (SSc)-associated PAH (SSc-PAH)) appear to have a similar pulmonary arterial and arteriolar remodelling pattern. Typical PAH lesions consist mainly of widely and uniformly distributed vascular alterations, including intimal fibrosis, and endothelial and smooth muscle cell proliferation, without obvious involvement of the bronchoalveolar architecture. These histological features are markedly different from pulmonary hypertension that arises as a consequence of chronic thromboembolic disease, chronic obstructive pulmonary disease, idiopathic pulmonary fibrosis and sarcoidosis, in which vascular occlusion develops as a consequence of emboli, arteriolar rarification, interstitial scarring or obstructing granulomas, respectively. Despite similarities in pathological anatomy, clinical management and outcome among the different forms of $\mathrm{PAH}$, there are, nonetheless, important differences that remain unexplained. In patients with SSc, PAH is a leading cause of mortality that requires intensive medical management. However, therapeutic responses are frequently less favourable compared to other forms of PAH [4-6].

\footnotetext{
*Univ Paris-Sud, Faculté de Médecine, Kremlin-Bicêtre, " AP-HP, Centre National de Référence de I'Hypertension Pulmonaire Sévère, Service de Pneumologie et Réanimation Respiratoire, Hôpital Antoine Béclère, Clamart, "INSERM U999, Hypertension Artérielle Pulmonaire, Physiopathologie et Innovation Thérapeutique, Clamart - Le Plessis Robinson, France.

CORRESPONDENCE: P. Dorfmüller, Centre National de Référence de l'Hypertension Artérielle Pulmonaire, Université Paris-Sud, INSERM U999, Service d'Anantomie et de Cytologie Pathologiques, Hôpital Marie Lannelongue, 133 Avenue de la Résistance, 92350 Le Plessis Robinson, France. E-mail: peter.dorfmuller@u-psud.fr
}

SSc-PAH treatment with vasodilators such as continuous intravenous epoprostenol is associated with improvements in exercise capacity and cardiopulmonary haemodynamics. However, therapeutic responses using prostanoid therapy in SSc-PAH patients are often disappointing, particularly compared to those with idiopathic PAH [5-8]. Similarly, endothelin receptor antagonists seem to show less impressive effects in SSc than in other forms of PAH $[9,10]$. In addition, therapeutic intervention in this population is potentially dangerous, with life-threatening acute pulmonary oedema a reported complication of vasoactive treatment in SSc-PAH [11]. Interestingly, these side-effects that occur in the context of treatment with vasodilators have been frequently described in the setting of another rare cause of pulmonary hypertension, pulmonary veno-occlusive disease (PVOD) [11, 12]. Pulmonary oedema and occult alveolar hemorrhage in treated PVOD patients is generally believed to occur as a result of fibrous intimal remodelling within small pulmonary venules that eventually leads to a post-capillary obstruction and transudation of fluid into alveoli, owing to the associated transcapillary haemodynamic gradient [12-15]. These observations have fuelled the hypothesis that venular remodelling may similarly characterise SSc-PAH and offer a potential explanation for the adverse therapeutic responses that have been previously reported in this disorder. A study by OvERBEEK et al. [16] in a recent issue of the European Respiratory Journal has shed additional light on the complex pathomechanisms of SSc-PAH. These authors analysed lung tissue from eight $\mathrm{PAH}$ patients with limited cutaneous SSc and compared finding to samples from 11 idiopathic PAH patients. Importantly, they found that all SScPAH patients displayed both arterial and venous remodelling, while venous lesions were present in only three of the 11 idiopathic PAH-patients. Of note, four of the SSc-PAH cases displayed a PVOD-like pattern, characterised by patchy capillary congestion and signs of occult alveolar haemorrhage. Similarly, our group has suggested a frequent PVOD-like involvement of the post-capillary vascular bed in the lungs of patients suffering from connective tissue disease-associated PAH [17]. Indeed, we found that six out of eight patients (four SSc, two lupus, one mixed connective tissue disease and one rheumatoid arthritis), and all four SSc-PAH patients, showed significant obstructive pulmonary vascular lesions predominantly involving the veins and pre-septal venules, as compared to five $(17 \%)$ out of 29 control PAH patients. Furthermore, lesions involving the small muscular arteries 
were consistently present in $\mathrm{PAH}$ associated with connective tissue disease, with intimal fibrosis and intraluminal thrombosis the most frequently identified pathological changes. Importantly, neither arterial nor venous remodelling was restricted to areas of interstitial fibrosis; indeed, these pathological vascular changes were also noted in regions showing otherwise normal bronchoalveolar architecture. In addition, the degree of interstitial lung disease (if any) did not correlate with the severity of the observed pulmonary vascular disease. While complex lesions are frequently observed in most other forms of $\mathrm{PAH}$, it is noteworthy that neither our group nor that of OVERBEEK et al. [16] has observed this histological entity in SSc-PAH. Although these morphological and clinical particularities are becoming increasingly recognised in connective tissue disease-associated $\mathrm{PAH}$, they remain poorly understood.

The systemic involvement of SSc also contributes to the unique characteristics observed in SSc-PAH, as distinct from idiopathic PAH. In this issue of the European Respiratory Journal, MATHAi et al. [18] have reported their findings from a study evaluating cardiac involvement in a cohort of SSc-PAH patients. Their results may in part explain the worse outcomes observed in this population. The authors compared serum levels of N-terminal pro-brain natriuretic peptide (NTproBNP), a circulating biomarker of neurohormonal activation produced and secreted by the myocardium, in 43 patients with idiopathic PAH and 55 SSc-PAH and correlated levels with haemodynamic parameters. They found that circulating NTproBNP levels were significantly increased in patients with SSc-PAH compared with idiopathic PAH patients, despite less severe haemodynamic impairment, as measured by right heart catheterisation. They also showed that haemodynamic indices closely correlate to NT-proBNP levels in SSc patients and that the latter parameter predicts survival. WILLIAMS et al. [19] have previously shown that increased NT-proBNP serum levels in connective tissue disease-associated $\mathrm{PAH}$ patients closely correlated with altered haemodynamics and reliably predicted survival. In considering the main findings of these studies, one may conclude that NT-proBNP expression is the cardiac consequence of increased pulmonary arterial pressures, but is more pronounced in SSc-PAH than in idiopathic PAH, even if haemodynamics are less severe in the former. One possible explanation for this seemingly contradictory conclusion is that SSc-PAH might differ in its pathological anatomy from other forms of PAH, as already suggested above. It is known that cardiac abnormalities other than those that develop as a consequence of pulmonary hypertension may be present in SSc patients. Fibrous scarring, probably due to micro-ischaemic and/or post-inflammatory events, is frequently found in the myocardium of the left ventricle, and chronic heart failure may occur in patients with scleroderma but lacking manifest PAH [20]. Although there is increasing interest in the potential role of NT-proBNP as a biomarker in PAH, this enzyme has long been known as an indicator of left heart failure [21, 22]. Interestingly, in a recent study by VON HAEHLING et al. [23], serum levels of NT-proBNP and several inflammatory markers (including tumour necrosis factor- $\alpha$, its receptors, and interleukin-10) from patients with right ventricular dysfunction due to chronic thrombembolic pulmonary hypertension were compared to patients with left ventricular dysfunction due to chronic heart failure. Although both groups showed significant increased levels of all markers, differences did not vary significantly between the different study groups. This finding suggests that different triggers may produce similar inflammatory signalling and pathological tissue reaction within the myocardium. One might then pose the question of whether elevated expression of NT-proBNP in SSc-PAH (as compared with idiopathic $\mathrm{PAH}$ ) is due, at least in part, to an underlying SSc-associated heart disease that involves the right and/or left ventricle and is particularly prone to increased pressure/shear stress. It is well established that a dysregulated fibrotic reaction in lung parenchyma, oesophagus and skin is characteristic of SSc, eventually leading to interstitial pulmonary fibrosis, oesophageal dysmotility/stenosis and scleroderma [24]. This impaired pro-fibrotic response to different stimuli has been attributed, in particular, to altered transforming growth factor- $\beta$ pathway signalling, which induces fibroblast-activation, reduction of extracellular matrix degrading activities and the initiation of $\alpha$-smooth muscle actin expression [25, 26]. It is, therefore, conceivable that the observed fibrous remodelling within pulmonary veins and venules in SSc-PAH may develop in a similar fashion; that is, an altered fibrous reaction pattern of the post-capillary vasculature to increased downstream pressures.

However, together with fibrous interstitial and pulmonary vascular remodelling, inflammation is a key contributor in SScassociated pulmonary disease. Indeed, OvERBEEK et al. [16] also observed transmural vascular inflammatory infiltrates, mainly consisting of lymphocytes, in four SSc-PAH and two idiopathic PAH cases. In two SSc-PAH patients, vasculitis was present at the venular level. This finding supports the hypothesis that inflammation and immunity may represent a possible common denominator among the various forms of PAH [27, 28]. Although there is no role for anti-inflammatory or immunosuppressive agents in most forms of $\mathrm{PAH}$, beneficial effects with these forms of therapy have nevertheless been reported in small retrospective studies of patients suffering from connective tissue disease-associated PAH (systemic lupus erythematosus and mixed connective tissue disease, but not SSc) [29-31].

We have recently defined new clinical parameters for the noninvasive and early differentiation of PVOD from idiopathic $\mathrm{PAH}$, an important practical issue in view of the significant risk associated with vasodilator therapy in PVOD [12, 32, 33]. Indeed, lung biopsy is not recommended in $\mathrm{PAH}$ patients because of the unacceptably high associated risk of death [12]. In contradistinction to PAH, PVOD patients show characteristic changes of nodular ground-glass opacities, septal lines and lymph node enlargement on high-resolution computed tomography of the chest. In addition, PVOD patients typically demonstrate lower values for partial pressure of arterial oxygen, diffusing lung capacity of carbon monoxide and oxygen saturation nadir during the 6-min walk test, when compared to idiopathic PAH patients [32]. Given these observations, it may be of considerable clinical utility to determine diagnostic and, therefore, prognostic parameters in other PAH groups with possible pulmonary venous and cardiac involvement, as is the case in SSc [34].

Considering these new possibilities in PAH management, the present work of MATHAI et al. [18], and the recent report by OVERBEEK et al. [16] in the European Respiratory Journal offer additional insights into the morphological characteristics of 
PAH in patients with SSc. These efforts also highlight the importance of pulmonary arterial remodelling and other lesions beyond this frontier.

\section{SUPPORT STATEMENT}

This study has been supported in part by grants from Ministère de l'Enseignement Supérieur et de la Recherche and the Université ParisSud 11 (Paris, France).

\section{STATEMENT OF INTEREST}

A statement of interest for M. Humbert can be found at www.erj. ersjournals.com $/ \mathrm{misc} /$ statements.dtl

\section{REFERENCES}

1 Simonneau G, Robbins IM, Beghetti M, et al. Updated clinical classification of pulmonary hypertension. J Am Coll Cardiol 2009; 54: Suppl. 1, S43-S54.

2 Humbert M, Sitbon O, Simonneau G. Treatment of pulmonary arterial hypertension. N Engl J Med 2004; 351: 1425-1436.

3 Humbert M, Simonneau G. Survival in patients with idiopathic, familial and anorexigen-associated pulmonary arterial hypertension in the modern management era. Eur Respir J 2009; 34: Suppl. 53,700 s.

4 Sanchez O, Humbert M, Sitbon O, et al. Treatment of pulmonary hypertension secondary to connective tissue diseases. Thorax 1999; 54: 273-277.

5 Kawut SM, Taichman DB, Archer-Chicko CL, et al. Hemodynamics and survival in patients with pulmonary arterial hypertension related to systemic sclerosis. Chest 2003; 123: 344-350.

6 Fisher MR, Mathai SC, Champion HC, et al. Clinical differences between idiopathic and scleroderma-related pulmonary hypertension. Arthritis Rheum 2006; 54: 3043-3050.

7 Badesch DB, Tapson VF, McGoon MD, et al. Continuous intravenous epoprostenol for pulmonary hypertension due to the scleroderma spectrum of disease. Ann Intern Med 2000; 132: 425-434.

8 Ramirez A, Varga J. Pulmonary arterial hypertension in systemic sclerosis: clinical manifestations, pathophysiology, evaluation, and management. Treat Respir Med 2004; 3: 339-352.

9 Denton CP, Humbert M, Rubin L, et al. Bosentan treatment for pulmonary arterial hypertension related to connective tissue disease: a subgroup analysis of the pivotal clinical trials and their open-label extensions. Ann Rheum Dis 2006; 65: 1336-1340.

10 Humbert M, Simonneau G. Drug insight: endothelin-receptor antagonists for pulmonary arterial hypertension in systemic rheumatic diseases. Nat Clin Pract Rheum 2005; 1: 93-101.

11 Humbert M, Sanchez O, Fartoukh M, et al. Short-term and longterm epoprostenol (prostacyclin) therapy in pulmonary hypertension secondary to connective tissue diseases: results of a pilot study. Eur Respir J 1999; 13: 1351-1356.

12 Montani D, Price LC, Dorfmuller P, et al. Pulmonary venoocclusive disease. Eur Respir J 2009; 33: 189-200.

13 Rabiller A, Jaïs X, Hamid A, et al. Occult alveolar haemorrhage in pulmonary veno-occlusive disease. Eur Respir J 2006; 27: 108-113.

14 Montani D, Jaïs X, Dorfmüller P, et al. Goal-oriented therapy in pulmonary veno-occlusive disease: a word of caution. Eur Respir J 2009; 34: 1204-1206.

15 Montani D, Jais X, Price LC, et al. Cautious epoprostenol therapy is a safe bridge to lung transplantation in pulmonary veno-occlusive disease. Eur Respir J 2009; 34: 1348-1356.
16 Overbeek MJ, Vonk MC, Boonstra A, et al. Pulmonary arterial hypertension in limited cutaneous systemic sclerosis: a distinctive vasculopathy. Eur Respir J 2009; 34: 371-379.

17 Dorfmüller P, Humbert M, Perros F, et al. Fibrous remodeling of the pulmonary venous system in pulmonary arterial hypertension associated with connective tissue diseases. Hum Pathol 2007; 38: 893-902.

18 Mathai SC, Bueso M, Hummers LK, et al. Disproportionate elevation of N-terminal pro-brain natriuretic peptide in scleroderma-related pulmonary hypertension. Eur Respir J 2010, 35: 95-104.

19 Williams MH, Handler CE, Akram R, et al. Role of N-terminal brain natriuretic peptide (N-TproBNP) in scleroderma-associated pulmonary arterial hypertension. Eur Heart J 2006; 27: 1485-1494.

20 Kahan A, Coghlan G, McLaughlin V. Cardiac complications of systemic sclerosis. Rheumatology (Oxford) 2009; 48: Suppl. 3, iii45iii48.

21 Gardner RS, Ozalp F, Murday AJ, et al. N-terminal pro-brain natriuretic peptide. A new gold standard in predicting mortality in patients with advanced heart failure. Eur Heart J 2003; 24: 17351743.

22 Moertl D, Berger R, Struck J, et al. Comparison of midregional proatrial and B-type natriuretic peptides in chronic heart failure: influencing factors, detection of left ventricular systolic dysfunction, and prediction of death. J Am Coll Cardiol 2009; 53: 1783-1790.

23 von Haehling S, von Bardeleben RS, Kramm T, et al. Inflammation in right ventricular dysfunction due to thromboembolic pulmonary hypertension. Int J Cardiol 2009; [Epub ahead of print DOI: 10. 1016/j.ijcard.2009.04.019].

24 Gabrielli A, Avvedimento EV, Krieg T. Scleroderma. N Engl J Med 2009; 360: 1989-2003.

25 Del Galdo F, Lisanti MP, Jimenez SA. Caveolin-1, transforming growth factor- $\beta$ receptor internalization, and the pathogenesis of systemic sclerosis. Curr Opin Rheumatol 2008; 20: 713-719.

26 Varga J, Pasche B. Transforming growth factor beta as a therapeutic target in systemic sclerosis. Nat Rev Rheumatol 2009; 5: 200-206.

27 Dorfmüller P, Perros F, Balabanian K, et al. Inflammation in pulmonary arterial hypertension. Eur Respir J 2003; 22: 358-363.

28 Hassoun PM, Mouthon L, Barbera JA, et al. Inflammation, growth factors, and pulmonary vascular remodeling. J Am Coll Cardiol 2009; 54: Suppl. 1, S10-S19.

29 Tanaka E, Harigai M, Tanaka M, et al. Pulmonary hypertension in systemic lupus erythematosus: evaluation of clinical characteristics and response to immunosuppressive treatment. J Rheumatol 2002; 29: 282-287.

30 Sanchez O, Sitbon O, Jaïs $X$, et al. Immunosuppressive therapy in connective tissue diseases-associated pulmonary arterial hypertension. Chest 2006; 130: 182-189.

31 Jais X, Launay D, Yaici A, et al. Immunosuppressive therapy in lupus- and mixed connective tissue disease-associated pulmonary arterial hypertension: a retrospective analysis of twenty-three cases. Arthritis Rheum 2008; 58: 521-531.

32 Montani D, Achouh L, Dorfmüller P, et al. Pulmonary venoocclusive disease: clinical, functional, radiologic, and hemodynamic characteristics and outcome of 24 cases confirmed by histology. Medecine (Baltimore) 2008; 87: 220-233.

33 Palazzini M, Manes A. Pulmonary veno-occlusive disease misdiagnosed as idiopathic pulmonary arterial hypertension. Eur Respir Rev 2009; 18: 177-180.

34 Vachiéry JL, Coghlan G. Screening for pulmonary arterial hypertension in systemic sclerosis. Eur Respir Rev 2009; 18: 162-169. 